\title{
Avaliação de pré-natal por indicador de qualidade
}

\section{Assessment of prenatal indicator for quality}

Camila Braga Derlan², Débora Cristina Haack Bassani ${ }^{1}$, Jéssica Chaves ${ }^{1}$, Luís Kanitz ${ }^{3}$, Thaíse Dipp ${ }^{3}$, Bianca Ghignatti ${ }^{4}$, Janine Koepp 5 , Lia Gonçalves Possuelo 6

1. Curso de Medicina da Universidade de Santa Cruz do Sul, Santa Cruz do Sul, RS, Brasil. 2. Curso de Farmácia da Universidade de Santa Cruz do Sul, Santa Cruz do Sul, RS, Brasil. 3. Curso de Enfermagem da Universidade de Santa Cruz do Sul, Santa Cruz do Sul, RS, Brasil. 4. Maternidade do Hospital Santa Cruz, Santa Cruz do Sul, RS, Brasil e Preceptora do Pro- Pet-Saúde Redes de Atenção Subprojeto Rede Cegonha. 5. Docente do Curso de Enfermagem da Universidade de Santa Cruz do Sul, Santa Cruz do Sul, RS, Brasil e Tutora do Pro- Pet-Saúde Redes de Atenção Subprojeto Rede Cegonha. 6. Docente do Departamento de Biologia e Farmácia da Universidade de Santa Cruz do Sul, Santa Cruz do Sul, RS, Brasil e Coordenadora do Pro- Pet-Saúde Redes de Atenção.

\section{Resumo}

Introdução: A constante avaliação da qualidade do pré-natal favorece a identificação de problemas de saúde e o desempenho da equipe de saúde. Objetivo: 0 objetivo do estudo foi avaliar a qualidade dos registros nas Carteiras das Gestantes que se internam na maternidade do Hospital Santa Cruz e, por meio desse registro, evidenciar a atenção pré-natal recebida pelas gestantes. Métodos: Trata-se de um estudo quantitativo e transversal. As informações foram coletadas das carteirinhas e dos prontuários das gestantes no período de janeiro a junho de 2014. Os dados foram coletados mediante a análise das carteiras de gestante, com aplicação de um instrumento padronizado (Formulário de Captação de dados). A análise e o processamento dos dados foram realizados no programa SPSS 22.0. Resultados: Houve o predomínio de mulheres entre 31 a 36 anos, 30,6\% (57) da amostra. A maioria iniciou o pré-natal no primeiro trimestre, com seis ou mais consultas. Os exames para hemoglobina e hematócrito alcançaram percentual de 34,9\% (65) correspondente a duas solicitações em momentos distintos, de acordo com o Programa de Humanização do Pré-natal e Nascimento. A glicemia em jejum, em sua maioria, foi requisitada em uma consulta. O VDRL foi solicitado em uma consulta assim como a urina tipo um, ficando abaixo do preconizado. 31,2\% (58) da amostra não haviam realizado os exames citados anteriormente. Conclusão: Com base neste estudo, pôde-se constatar que muitas carteiras provenientes do serviço básico de saúde necessitavam de melhor preenchimento, e que as gestantes que realizaram pré-natal em clínicas particulares possuíam dados insuficientes.

Palavras-chave: Cuidado pré-natal. Serviços de saúde. Indicadores básicos de saúde. Registros médicos.

\begin{abstract}
Introduction: The constant evaluation of the quality of prenatal care favors the identification of health problems and the performance of the health care team. Objective: The aim of this study was to evaluate the quality of the records in the portfolios of the pregnant women that been hospitalized at the Hospital Santa Cruz and through this record, highlight the prenatal care received by pregnant women. Methods: This is a quantitative and cross-sectional study and the information was collected from stamped certifications and records of pregnant women in the period from January to June 2014. Data were collected by analyzing the pregnant woman's license, applying for a standardized instrument (data capture form). The analysis and data processing were performed by means of the use of the SPSS 22.0 program. Results: There was a predominance of women between 31 and 36 years, 30.6\% (57) of the sample, most of whom began prenatal care in the first quarter, with six or more visits. Tests for hemoglobin and hematocrit percentage reached $34.9 \%$ (65) corresponding to two requests at different times, according to the Program of Humanization of Prenatal and Birth. The fasting glucose, mostly, was requested in a query. VDRL was requested in a query as well as urinalysis one being below the recommended values. Approximately $31.2 \%$ (58) of the samples did not perform the tests mentioned above. Conclusion: Based on this study, it was possible to observe that many portfolios from the basic health services needed to be better filled, and that pregnant women who had received prenatal care in private clinics showed insufficient data.
\end{abstract}

Palavras-chave: Prenatal Care. Health services; Basic health indicators. Medical records.

\section{INTRODUÇÃO}

A atenção no pré-natal é reconhecida por meio de condutas que proporcionam acolhimento de forma qualificada e humanizada, de acesso facilitado, garantindo a promoção, a prevenção e a assistência da gestante e do recém-nascido. Considera-se um demonstrativo do Pacto da Atenção Básica do Sistema único de Saúde (SUS), envolvendo todas as ações que permeiam o serviço, refletindo a participação de outros programas de saúde que, em conjunto, buscam prevenir, diagnosticar e tratar eventos indesejáveis na gestação, no parto e no puerpério ${ }^{1,2}$. A assistência pré-natal pode contribuir para desfechos mais favoráveis ao permitir a detecção e o tratamento oportuno de afeç̧ões, além de controlar fatores de risco que trazem complicações para a saúde da mulher e do bebê $\hat{e}^{3}$.

A constante avaliação da qualidade do pré-natal favorece a identificação de problemas de saúde e o desempenho da equipe de saúde, estratégias que podem manter ou modificar para melhor qualidade da assistência ${ }^{4,5,6}$.

O Programa de Humanização no Pré-natal e Nascimento (PHPN) visa à análise das necessidades de atenção direcionadas à gestante, ao recém-nascido e à mulher no período pósparto ${ }^{7}$. Ele tem por objetivo diminuir as altas taxas de morbimortalidade materna e perinatal, tomando medidas que

Correspondência: Jéssica Chaves. Acadêmica do Curso de Medicina da Universidade de Santa Cruz do Sul, Santa Cruz do Sul, Rio Grande do Sul, Brasil. Rua Fernando Abott, 174 - CEP: 96810-072 - Santa Cruz do Sul - RS. Telefone: (51) 81734720. E-mail: jessykasacht@yahoo.com.br

Conflito de interesse: Não há conflito de interesse por parte de qualquer um dos autores.

Recebido em: 16 Out 2014; Revisado em: 24 Abr 2015; 5 Mai 2015; Aceito em: 7 Maio 2015. 
garantem a melhoria do acesso, da cobertura e da qualidade do acompanhamento pré-natal, da assistência ao parto, ao puerpério e ao neonatal reproduzindo as ações já adotadas pelo Ministério da Saúde (MS) na área de atenção à gestante, como os investimentos nas redes estaduais de assistência à gestação de alto risco e o pagamento de procedimentos específicos ${ }^{4}$. Os indicadores de processo do PHPN incluem: trimestre de início do acompanhamento; percentual de mulheres inscritas no pré-natal em relação ao número de nascidos vivos; percentual de mulheres com seis ou mais consultas de pré-natal, todos os exames básicos, consulta puerperal, teste anti-HIV e dose imunizante da vacina antitetânica8 .

A Carteira da gestante é utilizada como fonte de informação pelo fato de conter o registro das principais informações sobre o acompanhamento pré-natal, facilitando a comunicação entre os profissionais nos diferentes níveis de atenção ${ }^{9}$. A importância do correto preenchimento da Carteira de gestante permite que a unidade de referência receba todas as informações necessárias para um atendimento mais qualificado e em tempo hábil. Um dos maiores problemas elencados pela literatura sugerem o não cumprimento de normas pelos profissionais e o não adequado preenchimento dos registros, verificandose que as informações repassadas não estão relacionadas às necessidades da paciente $\mathrm{e}^{10,11}$. A ausência de informações sobre a saúde materno-infantil nos cartões pode expressar diretamente a qualidade dos serviços de assistência pré-natal ${ }^{11}$.

Com o propósito de avaliar a qualidade da atenção pré-natal, estudos têm utilizado o chamado Índice de Kessner, que combina o número de consultas e o período de início do prénatal. Gestantes com mais de cinco consultas realizadas e com início do acompanhamento até o quarto mês tem seu prénatal considerado como adequado; quando iniciado após o sétimo mês de gravidez e com o número de consultas menor do que quatro, o pré-natal é categorizado como inadequado; as demais gestantes têm sua assistência classificada como intermediária. Além dessa avaliação, são incluídos itens considerados procedimentos mínimos para a realização de um pré-natal adequado como: informação sobre a data da última menstruação, peso materno, medida da altura uterina, medida da pressão arterial, exame ginecológico, prescrição de vitaminas e ferro, orientação sobre amamentação, exame dos seios e prescrição da vacina antitetânica ${ }^{10,12}$.

O estudo visa avaliar a qualidade dos registros na Carteira das gestantes que se internaram na maternidade de um hospital no interior do estado do Rio Grande do Sul (RS) e evidenciar a assistência pré-natal recebida por elas.

\section{MÉTODOS}

Trata-se de um estudo quantitativo e transversal, realizado por meio da revisão das carteiras e dos prontuários das gestantes que se internaram na maternidade de um hospital no interior do estado do Rio Grande do Sul (RS), Brasil. O Hospital Santa
Cruz (HSC) é uma entidade filantrópica, sem fins lucrativos, que atende pacientes SUS. Em relação ao número de leitos disponíveis, temos, na maternidade, 35 leitos destinados ao atendimento ginecológico e obstétrico; na Unidade de Cuidados Intermediários, 6 leitos e, na UTI Neonatal, são 8 leitos próprios para o atendimento de neonatos. Em virtude dos serviços disponibilizados, o HSC tornou-se referência para o Vale do Rio Pardo, em atendimento a gestantes e crianças de alto risco.

A população do estudo foi composta por 220 puérperas, da região do Vale do Rio Pardo (RS), que receberam assistência prénatal na rede pública e privada do município, e atendimento ao parto na maternidade do Hospital Santa Cruz.

As informações foram coletadas das carteirinhas e prontuários de gestantes, no período de janeiro a junho de 2014, durante a internação dessas pacientes na maternidade do Hospital Santa Cruz. Os dados foram coletados mediante análise da carteira de gestante, com aplicação de um instrumento padronizado (Formulário de Captação de dados), especialmente elaborado para a obtenção das informações, seguindo os critérios de Kessner adaptado, como parâmetro avaliador. Fizeram parte dos dados coletados: identificação, escolaridade, histórico materno, início do pré-natal e número de consultas realizadas.

Contabilizaram-se os exames básicos preconizados pelo MS, como VDRL, urina tipo I e glicemia em jejum. A tipagem sanguínea e a hemoglobina (hematócrito) $(\mathrm{Hb} / \mathrm{Ht})$ também foram registradas. Verificou-se o indicador da realização de procedimentos do exame clínico e obstétrico de rotina como: idade gestacional, peso, pressão arterial sistêmica, altura uterina, batimentos cardiofetais e apresentação fetal.

Como os dados foram coletados diretamente do prontuário e das carteiras de gestante, sem questionamento direto, não houve a necessidade da assinatura do Termo de Consentimento Livre Esclarecido por parte das puérperas em estudo. No entanto, manteve-se o procedimento ético e legal ao manipular dados secundários, conforme preconiza a Resolução 196/96. A análise e o processamento dados foram realizados com o programa Statistical Package for the Social Sciences (SPSS) versão 22.0. As variáveis contínuas foram expressas como média e desvio padrão (DP), enquanto as categóricas foram expressas como frequência absoluta e relativa. O nível de significância adotado foi de $5 \%(p \leq 0,05)$.

O presente estudo foi aprovado pelo Comitê de Ética em Pesquisa da Universidade de Santa Cruz do Sul, sob o número de CAAE: 14624913.0 .0000 .5343 e parecer $n=236523$.

\section{RESULTADOS}

De um total de 220 carteiras de gestante analisadas, 71 mulheres possuíam entre 31 a 36 anos, perfazendo 32,3\% do grupo. Grande parte da amostra (48,6\%) representava mulheres solteiras. Em geral, as ocupações mais prevalentes 
foram estudantes (5,5\%) e Do Lar (19,1\%).

Em relação à escolaridade, a variável contemplada com maior percentual foi ensino médio completo (30\%), seguido do ensino fundamental incompleto $(27,7 \%)$, havendo apenas um caso de analfabetismo. Uma das variáveis mais significativas foi a classificação do serviço de escolha para a realização do prénatal, alcançando um percentual elevado para o serviço público $(60,9 \%)$ e as clínicas particulares $(39,1 \%)$. Os demais valores percentuais encontram-se classificados na tabela 1.

Tabela 1. Características sociodemográficas de puérperas com carteira de gestantes internadas em um hospital do interior do estado do RS, Brasil, 2014.

\begin{tabular}{|c|c|c|c|}
\hline Variáveis sociodemográficas & № & $\%$ & DP \\
\hline \multicolumn{4}{|l|}{ Faixa etária } \\
\hline 13 a 18 & 18 & 8,2 & \\
\hline 19 a 24 & 48 & 21,8 & 1,184 \\
\hline 25 a 30 & 51 & 23,2 & \\
\hline 31 a 36 & 71 & 32,3 & \\
\hline$\geq 37$ & 32 & 14,5 & \\
\hline \multicolumn{4}{|l|}{ Escolaridade } \\
\hline Analfabetismo & 1 & 0,6 & \\
\hline Ensino fundamental incompleto & 61 & 27,7 & 1,811 \\
\hline Ensino fundamental Completo & 9 & 4,1 & \\
\hline Ensino médio incompleto & 16 & 7,3 & \\
\hline Ensino médio completo & 66 & 30,0 & \\
\hline Ensino superior incompleto & 25 & 11,4 & \\
\hline Ensino superior completo & 42 & 19,1 & \\
\hline \multicolumn{4}{|l|}{ Estado civil } \\
\hline Solteira & 107 & 48,6 & \\
\hline Casada & 70 & 31,8 & 1,089 \\
\hline Separada/Divorciada & 6 & 2,7 & \\
\hline União estável & 35 & 15,9 & \\
\hline Não consta & 1 & 0,5 & \\
\hline \multicolumn{4}{|l|}{ Ocupação } \\
\hline Do lar & 42 & 19,1 & \\
\hline Estudante & 12 & 5,5 & 19,396 \\
\hline Serviços gerais & 8 & 3,6 & \\
\hline Outros* & 158 & 71,8 & \\
\hline \multicolumn{4}{|l|}{ Convênio } \\
\hline SUS & 134 & 60,9 & \\
\hline $\mathrm{P}$ rivado & 86 & 39,1 & 2,695 \\
\hline
\end{tabular}

* Demais ocupações não foram descritas por apresentarem-se menos frequentes.

Em se tratando do número de consultas e do período de início do pré-natal, 165 (75,0\%) gestantes realizaram seis ou mais consultas, com início antes da 14 a semana gestacional (Tabela 2). Desse modo, o índice de Kessner resultou na maior proporção de casos na categoria adequado. As pacientes que realizaram menos de três consultas totalizaram 3 (1,3\%).

Tabela 2. Período gestacional de início do pré-natal de puérperas e número de consultas pré-natais.

\begin{tabular}{lrrrrr}
\hline & \multicolumn{3}{c}{ Número de consultas } & \multicolumn{2}{c}{$\begin{array}{l}\text { Valor } \\
\mathbf{p}\end{array}$} \\
\cline { 2 - 6 } $\begin{array}{l}\text { Início do } \\
\text { pré-natal }\end{array}$ & $\begin{array}{c}\geq 6 \\
\text { consultas }\end{array}$ & $\begin{array}{c}4-5 \\
\text { consultas }\end{array}$ & $\begin{array}{c}<4 \\
\text { consultas }\end{array}$ & Total & \\
\hline$<14$ semanas & 165 & 7 & 0 & 172 & \\
14-27 semanas & 32 & 9 & 2 & 43 & $<0,001$ \\
$>27$ semanas & 1 & 3 & 1 & 5 & \\
Total & 198 & 19 & 3 & 220 & \\
\hline
\end{tabular}

A tabela 3 avalia os exames complementares básicos realizados conforme o período da consulta pré-natal. Os exames solicitados na primeira consulta pré-natal e recomendados pelo MS foram ABO-Rh (87,3\%), Hb/Ht (27,3\%), glicemia de jejum (40,0\%), $\operatorname{VDRL}(51,8 \%)$ e Urina tipo 1 (40,0\%). 33,6\% da amostra não realizaram nenhum dos exames que garantissem a qualidade do pré-natal.

Tabela 3. Exames complementares básicos realizados conforme consulta pré-natal.

\begin{tabular}{|c|c|c|c|c|c|c|c|c|c|c|}
\hline \multirow[t]{2}{*}{ EXAMES } & \multicolumn{2}{|c|}{ ABO-Rh } & \multicolumn{2}{|c|}{$\mathrm{Hb} / \mathrm{Ht}$} & \multicolumn{2}{|c|}{$\begin{array}{l}\text { Glicemia } \\
\text { de jejum }\end{array}$} & \multicolumn{2}{|c|}{ VDRL } & \multicolumn{2}{|c|}{$\begin{array}{l}\text { Urina } \\
\text { tipo } 1\end{array}$} \\
\hline & № & $\%$ & № & $\%$ & № & $\%$ & № & $\%$ & № & $\%$ \\
\hline $\begin{array}{c}1 \underline{a} \\
\text { consulta }\end{array}$ & 192 & 87,3 & 60 & 27,3 & 88 & 40,0 & 114 & 51,8 & 88 & 40 \\
\hline $\begin{array}{c}2^{a} \\
\text { consulta }\end{array}$ & 13 & 5,9 & 81 & 36,8 & 75 & 34,1 & 65 & 29,5 & 63 & 28,6 \\
\hline $\begin{array}{c}3^{3 a} \\
\text { consulta }\end{array}$ & 2 & 0,9 & 59 & 26,8 & 36 & 16,4 & 31 & 14,1 & 38 & 38 \\
\hline $\begin{array}{c}4 \underline{a} \\
\text { consulta }\end{array}$ & 2 & 0,9 & 9 & 4,1 & 3 & 3,0 & 1 & 0,5 & 5 & 5 \\
\hline $\begin{array}{c}5^{a} \\
\text { consulta }\end{array}$ & - & - & 1 & 0,5 & - & 0 & - & & - & - \\
\hline $\begin{array}{c}\text { 6a } \\
\text { consulta }\end{array}$ & 11 & 5,0 & 10 & 4,5 & 18 & 8,2 & 9 & 4,1 & 26 & 26 \\
\hline p-Valor & & 0,001 & & 0,210 & & 0,676 & & 0,107 & & \\
\hline
\end{tabular}

$\mathrm{Na}$ tabela 4, verificou-se o número de vezes da realização de procedimentos do exame clínico e obstétrico, como idade gestacional (IG), peso, pressão arterial sistêmica (PA), altura uterina (AU), batimentos cardiofetais (BCF) e apresentação fetal, obtendo-se bom nível de significância para a maioria das variáveis. Das 220 mulheres, apenas 78 (35,5\%) estavam imunizadas para o tétano, e as 142 (64,5\%) restantes não apresentavam registro nas carteirinhas.

O preenchimento do exame para toxoplasmose foi realizado em 199 (90,5\%) carteirinhas. A imunização para rubéola mantevese baixa ou não foi registrada sua efetivação. 132 (60,0\%) mulheres tinham suas carteirinhas com espaço em branco neste quesito e, às vezes, sem discriminação junto das outras vacinas. 
Tabela 4. Procedimentos clínico-obstétricos essenciais de acordo com a consulta pré-natal.

\begin{tabular}{|c|c|c|c|c|c|c|c|c|c|c|c|c|}
\hline \multirow{2}{*}{ Procedimentos } & \multicolumn{2}{|c|}{ IG } & \multicolumn{2}{|c|}{ Peso materno } & \multicolumn{2}{|c|}{ PA } & \multicolumn{2}{|c|}{$A U$} & \multicolumn{2}{|c|}{$\mathrm{BCF}$} & \multicolumn{2}{|c|}{ Apresentação fetal } \\
\hline & $\mathbf{N}$ & $\%$ & $\mathbf{N}$ & $\%$ & $\mathbf{N}$ & $\%$ & $\mathbf{N}$ & $\%$ & $\mathbf{N}$ & $\%$ & $\mathbf{N}$ & $\%$ \\
\hline 1a consulta & 2 & 0,9 & 4 & 1,8 & 1 & 0,5 & 5 & 2,3 & 2 & 0,9 & 15 & 6,8 \\
\hline $2^{\mathrm{a}}$ consulta & 1 & 0,5 & 7 & 3,2 & 4 & 1,8 & 13 & 5,9 & 6 & 2,7 & 18 & 8,2 \\
\hline 3a consulta & 6 & 2,7 & 14 & 6,4 & 15 & 6,8 & 19 & 8,6 & 19 & 8,6 & 20 & 9,1 \\
\hline 4a consulta & 11 & 5 & 22 & 10 & 12 & 5,5 & 41 & 18,6 & 41 & 18,6 & 19 & 8,6 \\
\hline 5o consulta & 16 & 7,3 & 16 & 7,3 & 18 & 8,2 & 36 & 16,4 & 47 & 21,4 & 10 & 4,5 \\
\hline 6a consulta & 43 & 19,5 & 29 & 13,2 & 31 & 14,4 & 44 & 20 & 47 & 21,4 & 10 & 4,5 \\
\hline Consultas adicionais & 137 & 62,3 & 125 & 56,8 & 137 & 62,3 & 58 & 26,4 & 58 & 26,4 & 14 & 6,4 \\
\hline Nenhuma consulta & 4 & 1,8 & 3 & 1,4 & 2 & 0,9 & 4 & 1,8 & - & - & 114 & 51,8 \\
\hline p-Valor & & 0,895 & & $<0,001$ & & $<0,001$ & & $<0,001$ & & $<0,001$ & & 0,568 \\
\hline
\end{tabular}

Outros testes também foram realizados e podem ser observados na tabela 5.

Tabela 5. Frequência de puérperas que realizaram vacinas e sorologias durante o pré-natal.

\begin{tabular}{crrrrr}
\hline \multirow{2}{*}{$\begin{array}{c}\text { Frequência de } \\
\text { Puérperas }\end{array}$} & \multicolumn{2}{c}{ Sim } & \multicolumn{2}{c}{ Não } & DP \\
\cline { 2 - 5 } & $\mathbf{N}$ & $\%$ & $\mathbf{N}$ & $\%$ & \\
\hline Toxoplasmose & 199 & 90,5 & 21 & 9,5 & 0,295 \\
Rubéola & 88 & 40 & 132 & 60 & 0,801 \\
HBsAg & 175 & 79,5 & 45 & 20,5 & 0,404 \\
Anti-HBs & 53 & 24,1 & 167 & 75,9 & 0,429 \\
Anti-HCV & 118 & 53,6 & 101 & 45,9 & 0,585 \\
Anti-HIV & 204 & 92,7 & 16 & 7,3 & 0,26 \\
\hline
\end{tabular}

\section{DISCUSSÃO}

A avaliação de indicadores de saúde é uma ferramenta de suma importância para o acompanhamento de atividades e o impacto que elas fornecem quanto à relevância e à efetividade. A análise de carteiras de gestante conduz ao reconhecimento das ações que estão sendo realizadas e o efeito refletido no município quanto às taxas de mortalidade perinatal. Segundo dados oficiais da Secretaria Estadual de Saúde do RS, em 2012, foram 1489 mortes de crianças menores de um ano. Na região de cobertura da $13^{\circ}$ Coordenadoria Regional de Saúde, ocorreram 20 mortes e, destas, 17 eram de Santa Cruz do Sul.

Os índices mais baixos de mortalidade infantil e mortalidade materna foram registrados em 2013 . Em relação à mortalidade infantil foram 10,5 óbitos por mil nascidos vivos e a mortalidade materna com 29,7 óbitos maternos a cada cem mil crianças nascidas vivas. A morte de mulheres durante a gravidez, 0 parto e o puerpério, no mesmo período, foi um total de 107 óbitos. Deste índice, as quatro mortes que representaram a $13^{\circ}$ Coordenadoria Regional de Saúde ocorreram nos município de Sinimbu, Venâncio Aires e Vera $\mathrm{Cruz}^{13}$.
Observou-se predomínio de mulheres entre 31 a 36 anos, perfazendo $32,3 \%$ do grupo. Os dados apontados não são compatíveis com outros estudos já realizados que caracterizam um percentual mais elevado para gestantes adolescentes ${ }^{14-17}$. $\mathrm{Na}$ amostra, observou-se maior frequência de pacientes com ensino médio completo. Segundo estudos realizados de uma amostra de 75 mulheres, 58,6\% possuíam entre cinco e oito anos de estudo, e 25,3\%, acima de oito anos, sem ocorrência de ensino superior ${ }^{14}$.

Na pesquisa, pôde-se identificar que grande parte da amostra iniciou o pré-natal logo no primeiro trimestre e realizou seis ou mais consultas de acompanhamento, caracterizando a assistência como adequada. Já informações da literatura nacional revelam que a baixa cobertura de consultas no prénatal mantém-se prevalente, assim, como problemas em sua distribuição, conforme o calendário preconizado pelo $\mathrm{MS}^{18,19}$.

Segundo o PHPN, por meio da Portaria GM n 569, de 1/6/2000 do $M S$, a conclusão da assistência pré-natal se dá com, no mínimo, seis consultas de pré-natal, a realização de todos os exames obrigatórios: um para tipagem, hemoglobina e hematócrito $(\mathrm{Hb} / \mathrm{Ht})$ e dois para VDRL, urina e glicemia em jejum, imunização antitetânica e, após o parto, a realização da primeira consulta de pré-natal ${ }^{20}$.

Deve-se preconizar a solicitação de todos esses exames já na primeira consulta, com realização durante o primeiro trimestre de gestação e repetição do VDRL, urina tipo 1 e glicemia de jejum por volta da 28 a semana ${ }^{4,14}$. Nota-se que a preocupação primordial está relacionada ao registro dos resultados e não ao período gestacional de solicitação ou realização dos exames ${ }^{21}$.

Foram estabelecidos pontos de corte, considerando pré-natal adequado com, no mínimo, seis consultas realizadas. Destas, cinco apresentavam registros para altura uterina, idade gestacional, pressão arterial e peso; registro igual ou maior a quatro para batimentos cardiofetais e apresentação fetal com dois ou mais registros. A mesma autora também encontrou altas taxas de sub-registros para a apresentação fetal e a vacina 
antitetânica, como no presente estudo7.

Durante a análise do estudo, observou-se que a tipagem sanguínea foi realizada em grande percentual, apenas uma vez, alcançando o almejado. Os exames para hemoglobina e hematócrito alcançaram $36,8 \%$ correspondentes a duas solicitações em momentos distintos, de acordo com o PHPN. Na avaliação dos procedimentos clínicos e obstétricos, priorizouse a verificação - em grande parte das consultas - do peso materno, da pressão arterial e do cálculo da idade gestacional. Os registros com menor índice foram medida da altura uterina, BCFs e apresentação fetal. Já outros estudos indicam que, no pré-natal, como os procedimentos realizados nas consultas não demandam uma estrutura sofisticada de atendimento e dependem, quase exclusivamente, da atuação dos recursos humanos, os profissionais envolvidos com o cuidado pré-natal valorizam a realização desses procedimentos e compreendem sua importância para a qualidade da assistência ${ }^{22,23}$.

Nos campos de registro que se apresentaram em branco, considerou-se a não realização do procedimento, assim como foi evidenciado em outros estudos que analisaram o mesmo parâmetro ${ }^{21}$. Foram muitas carteirinhas não preenchidas, o que dificultou sua análise. A assistência provavelmente foi realizada, mas, sem o registro, a avaliação quanto à qualidade ficou comprometida. Portanto, não pôde ser realizada.

Por meio da avaliação nas carteiras, podem ser identificados erros ou deficiência na assistência pré-natal que resultam na elevação dos dados já citados ou permitem que condutas sejam tomadas e falhas evitadas. A análise dos registros do médico, obtidos durante a consulta, são os mais importantes. Porém, são os dados que mais se mostram ausentes. Ressalta-se que tais informações são meramente informativas, não constando nas carteirinhas de pré-natal. Todos os dados informativos foram retirados do prontuário ou da Declaração de Nascido Vivo do recém-nascido.

A vacina antitetânica está disponível na maioria dos serviços públicos de saúde, porém o tétano e suas possíveis complicações não são de conhecimento da maioria das gestantes, independentemente do serviço em que o controle de pré-natal é realizado ${ }^{24}$. O preenchimento do exame para toxoplasmose foi realizado em 199 (90,5\%) carteirinhas, demonstrando o reconhecimento por parte dos profissionais diante de sua importância. Em estudos analisados, o exame sorológico para toxoplasmose foi realizado, pelo menos, uma vez em 392 gestantes, de um total de 449. As mulheres que não realizaram o exame não sabiam se houve solicitação médica para sua realização ${ }^{21}$. A infecção fetal apresenta um risco dependente da idade gestacional, e, por isso, são de suma importância as medidas que evitam a transmissão; é durante as consultas de pré-natal que essas informações podem ser repassadas às gestantes.

No primeiro trimestre de gestação, há $13 \%$ de chances de lesões graves e $87 \%$ de lesões clinicamente leves; no segundo trimestre, a probabilidade altera para $10 \%$ e $90 \%$ respectivamente e, no último trimestre, as lesões podem estar ausentes ${ }^{25}$.

O acompanhamento de pré-natal previne muitas complicações, e o correto preenchimento das carteiras facilita o trabalho de assistência da unidade de referência. Com base neste pressuposto, pôde-se identificar que muitas carteiras provenientes do serviço básico de saúde demonstraram-se mais bem preenchidas, enquanto as gestantes que realizaram pré-natal em clínicas particulares demonstraram dados insuficientes, dando margem a críticas e dúvidas recebidas na assistência de pré-natal.

Os exames de VDRL, urina tipo um e glicemia em jejum não alcançaram o preconizado pelo Ministério da Saúde. Da mesma forma, a imunização para rubéola e o Anti-Hbs e Anti-HCV apresentaram baixo registro nos cartões ou nas carteirinhas. Os fatores responsáveis por essa situação podem ser diversos: não realização do exame por escolha da gestante, realização do exame, mas sem retorno ao serviço de pré-natal, falta de solicitação ou falta de registro por parte do profissional médico e também ilegibilidade.

Apesar das diversas limitações do estudo, pudemos obter importantes considerações dispostas ao longo do artigo, encorajando mais estudos na área. Destaca-se que o $\mathrm{n}$ da amostra foi razoável, ainda que tenha havido um único centro estudado, bem como lembramos que as informações de análise do instrumento utilizado dependeram do profissional que o preenchia: no caso a carteira da gestante e o prontuário.

Muitos exames não datavam o trimestre de realização, o que dificultou a análise de dados e fez que a pesquisa se direcionasse para uma projeção quantitativa. Diante disso, novos esforços devem ser buscados para a adequação da assistência pré-natal em relação ao início da atenção, ao número de consultas e aos exames complementares básicos realizados. Em alguns casos, não se tinha a informação do local de realização do pré-natal. Portanto, é de suma importância que os profissionais de saúde envolvidos na assistência do pré-natal avaliem a paciente de forma integral e registrem suas práticas para melhorar o atendimento sucessor e torná-lo mais eficiente e qualificado.

\section{REFERÊNCIAS}

1. Ribeiro JM, Costa NR, Pinto LFS, Silva PL. Atenção ao pré-natal na percepção dos usuários do Sistema Único de Saúde: um estudo comparativo. Cad Saúde Pública. 2004 Mar-Apr; 20(2):534-45. doi: http://dx.doi.org/10.1590/S0102$311 \times 2004000200022$

2. Brasil. Ministério da Saúde. Secretaria de Atenção à Saúde. Departamento de Ações Programáticas Estratégicas. Área Técnica de Saúde da Mulher. Pré-natal e puerpério: atenção qualificada e humanizada - manual técnico. 3. ed. Brasília:

\section{Ministério da Saúde; 2006}

3. Domingues RMSM, Hartz ZMA, Dias MAB, Leal MC. Avaliação da adequação da assistência pré-natal na Rede SUS do município do Rio de Janeiro, Brasil. Cad. Saúde Pública. 2012; 28(3):425-437.

4. Schirmer J, Parras AP, Leocádio E, Fajardo ML, Reis PAK, Chacel PP, et al. Assistência pré-natal: manual técnico. 3.ed. Brasília: Secretaria de Políticas de 
Saúde/Ministério da Saúde, 2000.

5. Coimbra LC, Silva AAM, Mochel EG, Alves MTSSB, Ribeiro VS, Aragão VMF. et al. Fatores associados à inadequação do uso da assistência pré-natal. Rev Saúde Pública. 2003 Ago; 37(4): 456-62. doi: http://dx.doi.org/10.1590/S003489102003000400010 .

6. Ministério da Saúde, Secretaria de Atenção à Saúde, Departamento de Ações Programáticas Estratégicas. Gestação de alto risco: manual técnico. 5.ed. Brasília: Ministério da Saúde; 2010. Série A

7. Anversa ETR, Bastos GAN, Nunes LN, Dal Pizzol Tda S. [Quality of prenatal care: traditional primary care and Family Health Strategy units in a city in southern Brazil]. Cad. Saúde Pública. 2012 abr; 28(4): 789-800. PubMed PMID: 22488324.

8. Andreucci CB, Cecatti JG, Macchetti CE, Sousa MH. Sisprenatal como instrumento de avaliação da qualidade da assistência à gestante. Rev Saúde Pública. 2011 Out; 45(5):854-63. doi: http://dx.doi.org/10.1590/S003489102011005000064 .

9. Polgliani RBS, Neto ETS, Zandonade E. Informações dos cartões de gestantes e dos prontuários da atenção básica sobre assistência pré-natal. Rev Bras Ginecol Obstet. 2014; 36(6):269-75. doi: http://dx.doi.org/10.1590/S0100720320140004907

10. Silveira DS, Santos IS. Dias-da-Costa JS. [Prenatal care at the primary health care level: an assessment of the structure and process]. Cad. Saúde Pública. 2001 Jan-Feb; 17(1): 131-139. doi: http://dx.doi.org/10.1590/S0102 $311 \times 2001000100013$.

11. Santos ET, Neto, Oliveira AE, Zandonade E, Gama SGN, Leal MC. O que os cartões de pré-natal das gestantes revelam sobre a assistência nos serviços do SUS da Região Metropolitana da Grande Vitória, Espírito Santo, Brasil? Cad. Saúde Pública. 2012 Set; 28(9):1650-1662. doi: http://dx.doi.org/10.1590/ S0102-311X2012000900005.

12. Takeda S. Avaliação de unidade de atenção primária: modificação dos indicadores de saúde e qualidade da atenção [dissertação]. Pelotas (RS): Universidade Federal de Pelotas; 1993.

13. Secretaria Estadual da Saúde. Mortalidade mulheres gravidez, parto e puerpério. Dados e Estatística. Disponível em: <http://www.saude.rs.gov.br/ lista/586/2012_-_Mortalidade_geral> Acessado em: 12/06/14.

14. Jandrey CM. Avaliação da Qualidade do Processo de Assistência Pré-natal na Rede Pública do Município de Cachoeirinha/RS [dissertação]. Porto Alegre (RS): Universidade Federal do Rio Grande do Sul; 2005.

15. Dias-da-Costa JS, Madeira ACC, Luz RM, Britto MAP. Auditoria médica: programa de pré-natal em posto de saúde na região Sul do Brasil . Rev. Saúde Pública. 2000 Ago; 34(4): 329-360. doi: http://dx.doi.org/10.1590/S003489102000000400003.

16. Coutinho T, Teixeira MT, Dain S, Coutinho LM. Adequação do processo de assistência pré-natal entre as usuárias do Sistema Único de Saúde em Juiz de Fora-MG. Rev. Bras. Ginecol Obstet. 2003 Nov- Dez; 25(10): 717- 724. doi: http://dx.doi.org/10.1590/S0100-72032003001000004.

17. Brasil, Ministério da Saúde, Secretaria de Vigilância em Saúde, Departamento de Análise de Situação de Saúde. Saúde Brasil 2004- uma análise da situação de saúde. Brasília: Ministério da Saúde; 2004.

18. Andreucci CB, Cecatti, JG. Desempenho de indicadores de processo do Programa de Humanização do Pré-Natal e Nascimento no Brasil: uma revisão sistemática. Cad Saúde Pública. 2011 Jun; 27(6):1053-64. doi: http://dx.doi. org/10.1590/S0102-311X2011000600003.

19. Chrestani MAD, Santos IS, Cesar JA, Winckler LS, Gonçalves TS, Neumann NA. Assistência à gestação e ao parto: resultados de dois estudos transversais em áreas pobres das regiões Norte e Nordeste do Brasil. Cad Saúde Pública. 2008 Jul; 24(7):1609-18. doi: http://dx.doi.org/10.1590/S0102-311X2008000700016.

20. BRASIL. Ministério da Saúde. Secretaria de Políticas de Saúde. Humanização do parto. Programa de Humanização no pré-natal e nascimento. Brasília: DF; 2002. Disponível em: http://bvsms.saude.gov.br/bvs/publicacoes/parto.pdf. Acessado em: 12/06/14.

21. Kawasaki ML, Carvalho PN, Lucarevschi BR. Atenção à toxoplasmose durante a gestação em população carente do interior do Estado de São Paulo. Pediatria (São Paulo). 2006; 28(4):242-50.

22. Carvalho DS, Novaes HMD. Avaliação da implantação de programa de atenção pré-natal no município de Curitiba, Paraná, Brasil: estudo em corte de primigestas. Cad Saúde Pública. 2004; 20(Suppl 2):S220-S230.

23. Koffman MD, Bonadio IC. Avaliação da atenção pré-natal em uma instituição filantrópica da cidade de São Paulo. Rev. Bras. Saúde Matern. Infant. 2005 Dez; 5 (Supl 1): S23-S32. doi: http://dx.doi.org/10.1590/S1519-38292005000500003.

24. Mattos LMBB, Caiaffa WT, Bastos RR, Tonelli E. Oportunidades perdidas de imunização antitetânica de gestantes de Juiz de Fora, Minas Gerais, Brasil. Rev Panam Salud Publica. 2003 Nov;14(5):350-4. doi: http://dx.doi.org/10.1590/ S1020-49892003001000010.

25. Silva LR, Okasaki ELFJ. Enfermagem e a prevenção da Toxoplasmose durante a gestação. Rev. Enferm. UNISA. 2012; 13(1):43-7.

\section{Como citar este artigo/How to cite this article:}

Bassani DC, Derlan CB, Chaves J, Kanitz L, Dipp T, Ghignatti B, Koepp J, Possuelo L. Avaliação de pré-natal por indicador de qualidade. J Health Biol Sci. 2015 Abr-Jun; 3(2):67-72. 